\title{
Resistência ao Fluxo de Gases Através das Cânulas de Intubação de Dupla Luz *
}

\section{Airflow Resistance of Double-Lumen Tracheal Tubes}

Klaus Carvalho Lustosa ${ }^{1}$, Eric Schalch ${ }^{1}$, Joaquim Edson Vieira, TSA ${ }^{2}$, Fábio Ely Martins Benseñor ${ }^{3}$

\author{
RESUMO \\ Lustosa KC, Schalch E, Vieira JE, Benseñor FEM - Resistência \\ ao Fluxo de Gases Através das Cânulas de Intubação de Dupla \\ Luz
}

Justificativa e Objetivos - As cânulas de dupla luz podem impor resistências distintas ao fluxo. Esta disparidade pode acarretar ventilação pulmonar não homogênea. O objetivo deste trabalho é comparar o padrão resistivo da cânula de intubação de dupla luz 37 FR com o das cânulas convencionais, utilizadas em nosso meio, quando submetidas a fluxos diferentes.

Método - Foram utilizadas cânulas de intubação orotraqueal com diâmetros internos de 7, 7,5, 8 e 8,5 mm e de dupla luz 37 FR. Os fluxos foram gerados e mantidos por aparelho de anestesia. A pressão resistiva gerada nas cânulas foi aferida por um pneumotacógrafo de orifício variável e convertida para sistema digital. As resistências foram obtidas dividindo-se as pressões medidas pelos fluxos empregados. Os dados foram submetidos à análise de variância (ANOVA) e comparados pelo teste de Tukey.

Resultados - Foram registradas cinco medidas obtidas aleatoriamente. Todas as cânulas estavam abertas para o meio-ambiente. Resistências estão em $\mathrm{cmH}_{2} \mathrm{O} \cdot \mathrm{L}^{-1} \cdot \mathrm{s}^{-1}$. A resistência aumenta de forma linear com elevação do fluxo. $A$ cânula 37 FR dupla luz mostrou padrão resistivo semelhante ao da cânula com diâmetro interno de $7,5 \mathrm{~mm}$. A oclusão dos ramos da sonda 37 FR eleva significativamente a resistência ao fluxo.

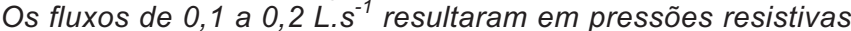
semelhantes para uma mesma sonda de intubação, exceto para cânula $7 \mathrm{~mm}$ ou para a $37 \mathrm{FR}$ com oclusão $(p<0,001)$.

Conclusões - Os padrões resistivos da cânulas 37 FR e 7,5 $\mathrm{mm}$ mostraram-se bastante próximos. A oclusão de algum ramo da sonda de dupla luz eleva significativamente a resistência, mas de modo indistinto entre ambos ramos para fluxos abaixo de 0,5 L.s ${ }^{-1}$. Os resultados do presente estudo sugerem a redução de fluxos inspiratórios como parâmetro para diminuir a resistência do sistema de ventilação, quando um dos lumens de um tubo de dupla luz for obstruído.

UNITERMOS: ANESTESIA; EQUIPAMENTOS: tubo traqueal de dupla luz

\footnotetext{
* Recebido do (Received from) Centro de Ensino e Treinamento da Disciplina de Anestesiologia da Faculdade de Medicina da Universidade de São Paulo. (HCFMUSP)

1. $M E_{2}$ do CET/SBA, HCFMUSP

2. Médico Assistente da Divisão de Anestesia, Professor Colaborador Disciplina de Clínica Geral do HCFMUSP

3. Médico Assistente da Divisão de Anestesia, Supervisor, Unidade de Apoio Cirúrgico (UAC), HCFMUSP

Apresentado (Submitted) em 03 de janeiro de 2002

Aceito (Accepted) para publicação em 25 de abril de 2002

Correspondência para (Mail to):

Dr. Joaquim Edson Vieira

Av. Dr. Arnaldo 455 sala 1216

01246-903 São Paulo, SP

E-mail: joaquimev@ hotmail.com

(c) Sociedade Brasileira de Anestesiologia, 2002
}

\author{
SUMMARY \\ Lustosa KC, Schalch E, Vieira JE, Benseñor FEM - Airflow Re- \\ sistance of Double-Lumen Tracheal Tubes
}

Background and Objectives - Double lumen tubes may determine different flow resistances. This disparity may result in non-homogeneous ventilation. This study aimed at comparing the resistive pressure of $37 \mathrm{FR}$ double lumen tubes to distinct flows as compared to conventional adult tracheal tubes.

Methods - Tracheal tubes with internal diameters of 7; 7.5; 8 and 8.5 millimeters $(\mathrm{mm})$ and $37 \mathrm{FR}$ double lumen tubes were tested. Flows were generated and maintained by a conventional anesthesia ventilator. Resistive pressure generated in the tubes was measured by a variable inlet pneumotachograph and converted to a digital system. Resistances were obtained by dividing measured pressures by flows. Data were submitted to analysis of variance (ANOVA) and Tukey's test.

Results - Five independent measurements were obtained. All tubes were opened to the environment. Resistances are shown in $\mathrm{cmH}_{2} \mathrm{O} \cdot \mathrm{L}^{-1} \cdot \mathrm{s}^{-1}$. Resistance is linearly increased with increased flow. The $37 \mathrm{FR}$ tube had a resistive pattern similar to the $7.5 \mathrm{~mm}$ tracheal tube. The occlusion of any double lumen branch significantly increases flow resistance. Flows set at 0.1 L. $s^{-1}$ to $0.2 L . s^{-1}$ resulted in similar resistive pressures for all tubes, except for the $7 \mathrm{~mm}$ or the occluded $37 \mathrm{FR}$ tube ( $p<$ $0.001)$.

Conclusions - Resistive patterns of $37 \mathrm{FR}$ and $7,5 \mathrm{~mm}$ tubes were very similar. Any double lumen branch occlusion significantly increases resistance, however in a similar way for both branches for flows below $0.5 \mathrm{~L} . \mathrm{s}^{-1}$. These results suggest the use of low inspiratory flow to minimize ventilatory system resistive pressure when any branch of a double-lumen tube is occluded.

KEY WORDS: ANESTHESIA; EQUIPMENTS: double lumen tracheal tube

\section{INTRODUÇÃO}

$\mathrm{O}$ grande progresso da cirurgia torácica atualmente se deve ao melhor entendimento da fisiologia do paciente com o tórax aberto. Nos primórdios da especialidade a barreira a ser transposta foi o acesso à cavidade torácica, uma vez que pneumotórax em um paciente em respiração espontânea pode causar a morte ${ }^{1}$. Esse problema foi resolvido com a introdução da ventilação mecânica com pressão positiva, através dos tubos traqueais de luz simples.

A técnica de ventilação monopulmonar com tubos de dupla luz permite melhor acesso à cavidade torácica. A utilização destes tubos permite melhor apresentação cirúrgica, facilitando o trabalho do cirurgião, diminuição do trauma pulmonar e melhores técnicas de assistência ventilatória dos pacientes admitidos nas unidades de terapia intensiva. No entanto, sua utilização apresenta desvantagens também, que são o trauma de traquéia, edema de laringe, hipoxemia se- 
cundária e o aumento excessivo da resistência ao fluxo aéreo ${ }^{2,3}$.

As cânulas de dupla luz tipo Robertshaw aparentemente têm menor resistência ao fluxo que suas correlatas, Carlens ou White. Ainda mais, estas últimas podem apresentar resistências distintas ao fluxo medido nos orifícios lateral e inferior do ramo direito das cânulas. Este tipo de disparidade pode, eventualmente, acarretar ventilação não homogênea ${ }^{4}$. No entanto, em situações clínicas, aparentemente não existe redução de fluxo para as cânulas de dupla luz atualmente utilizadas ${ }^{5}$.

Os filtros bacterianos são muitas vezes associados à utilização de cânulas de dupla luz, particularmente com o intuito de proteger os ramos do ventilador e monitores de contaminação por secreção oriunda das vias aéreas do paciente.

O objetivo deste trabalho é comparar o padrão resistivo da cânula de intubação de dupla luz 37 FR com as cânulas convencionais, utilizadas em nosso meio, quando submetidas a fluxos diferentes. Também serão observadas as propriedades resistivas do filtro bacteriano para uso em sonda de intubação orotraqueal.

\section{MÉTODO}

Foram utilizadas cânulas de intubação orotraqueal com diâmetros internos de 7, 7,5, 8 e 8,5 mm e de dupla luz 37 FR (Mallinckrodt, St. Louis, MO). O filtro disponibilizado foi o Thermovent BVF 100/584/100 (Portex, UK). Os fluxos foram gerados e mantidos pelo aparelho de anestesia Linea (Intermed, São Paulo, Brasil). A pressão resistiva gerada nas cânulas foi aferida por um pneumotacógrafo de orifício variável (Bicore CP100 Respiratory Monitor, Allied Healthcare, USA) conectado ao sistema circular do aparelho de anestesia.

O tubo de dupla luz permite que um ou ambos os pulmões possam ser ventilados simultânea ou separadamente, sendo utilizados em cirurgia torácica. A cânula testada tem o ramo esquerdo dirigida para o brônquio e o ramo direito traqueal. O material utilizado para sua construção é o mesmo plástico semi-rígido das cânulas de luz simples.
Os fluxos aplicados às cânulas foram previamente calibrados para uma referência padrão (Timeter RT200, Allied Healthcare, USA) e situados em 0,1 litros por segundo (L.S $\left.{ }^{-1}\right)$, $0,2,0,3,0,5$ e $0,83 \mathrm{~L} . \mathrm{S}^{-1}$ (respectivamente $6,12,18,30$ e 50 litros por minuto). Os dados analógicos adquiridos do pneumotacógrafo por um sensor (Var-Flex, Allied Healthcare, USA) foram convertidos para o sistema digital pela utilização de uma placa A/D numa freqüência de $200 \mathrm{~Hz}$ e de seu software Aqdados e armazenados em computador pessoal. As resistências foram obtidas dividindo-se as pressões medidas pelos fluxos empregados. Foram comparadas as resistências para diferentes fluxos em cânulas de mesmo diâmetro e para o mesmo fluxo entre diferentes tubos. Os dados foram submetidos à análise de variância (ANOVA) e comparados pelo teste de Tukey. Foi considerado $p<0,05$ como significativo entre os grupos, para $\alpha=0,05$ e $\beta=0,80$.

\section{RESULTADOS}

As resistências foram registradas em cinco medidas obtidas aleatoriamente para cânulas e fluxos. Todas as cânulas estavam abertas para o meio-ambiente. A de dupla luz 37 FR foi testada para ambas as vias, simultaneamente, ou para cada uma isoladamente com seu ramo lateral ocluído. A tabela I mostra as médias e os desvios padrões das resistências obtidas para resistências em $\mathrm{cmH}_{2} \mathrm{O} \cdot \mathrm{L}^{-1} \cdot \mathrm{s}^{-1}$.

Acânula $37 \mathrm{FR}$ dupla luz mostrou padrão resistivo semelhante ao da cânula com diâmetro interno de $7,5 \mathrm{~mm}$ para todos os fluxos testados, porém sem significância estatística $(2,00 \pm$ 0,08 vs $2,18 \pm 0,12$, Fluxo de 0,1 L.s $^{-1} ; 1,88 \pm 0,13$ vs $2,47 \pm$ $0,07,0,2$ L.s $^{-1} ; 3,61 \pm 0,39$ vs $3,45 \pm 0,36,0,3$ L. $^{-1} ; 4,78 \pm 0,17$ vs $4,60 \pm 0,15,0,5{\mathrm{~L} . \mathrm{s}^{-1}}^{-1}$ e $6,58 \pm 0,31$ vs $7,02 \pm 0,47,0,83$ L. $\mathrm{s}^{-1}$ ). A resistência aumenta de forma linear com elevação do fluxo, mas se reduz em cânulas de maior diâmetro interno submetidas ao mesmo fluxo. A oclusão dos ramos da sonda 37 FR eleva significativamente sua resistência. As resistências apresentadas pelo filtro foram crescentes com aumento do fluxo, porém sem significância estatística. No entanto, o

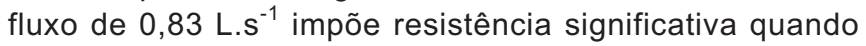
comparada às oferecidas pelo filtro sob menores fluxos $(p<$ 0,001).

Tabela I - Resistência à Passagem de Diferentes Fluxos de Gases pelas Cânulas Estudadas (Média \pm DP)

\begin{tabular}{|c|c|c|c|c|c|}
\hline \multirow[b]{2}{*}{ Cânulas } & \multicolumn{5}{|c|}{ Fluxos $\left(\right.$ L.s $\left.^{-1}\right)$} \\
\hline & 0,1 & 0,2 & 0,3 & 0,5 & 0,83 \\
\hline $7 \mathrm{~mm}$ & $2,40(0,09)$ & $3,29(0,17)$ & $4,01(0,24)$ & $5,62(0,06)$ & $8,51(0,20)$ \\
\hline $7,5 \mathrm{~mm}$ & $2,18(0,12)$ & $2,47(0,07)$ & $3,45(0,36)$ & $4,60(0,15)$ & $7,02(0,47)$ \\
\hline $8 \mathrm{~mm}$ & $1,47(0,21)$ & $1,68(0,09)$ & $2,46(0,15)$ & $2,98(0,18)$ & $4,66(0,37)$ \\
\hline $8,5 \mathrm{~mm}$ & $0,80(0,17)$ & $0,75(0,08)$ & $1,81(0,17)$ & $2,31(0,39)$ & $3,54(0,23)$ \\
\hline $37 \mathrm{FR}$ & $2,00(0,08)$ & $1,88(0,13)$ & $3,61(0,39)$ & $4,78(0,17)$ & $6,58(0,31)$ \\
\hline $37 \mathrm{DO}$ & $5,28(0,26)$ & $7,75(0,36)$ & $10,33(0,90)$ & $14,09(0,49)$ & $20,04(0,48)$ \\
\hline $37 \mathrm{EO}$ & $5,28(0,36)$ & $7,97(0,21)$ & $10,76(0,94)$ & $14,81(0,47)$ & $21,58(1,04)$ \\
\hline Filtro & $1,60(0,06)$ & $1,72(0,11)$ & $1,81(0,09)$ & $1,72(0,05)$ & $2,09(0,09)$ \\
\hline
\end{tabular}


A oclusão dos ramos gerou aumento linear mais acentuado da resistência, ainda proporcional ao fluxo. No entanto, não se observaram diferenças significativas entre os ramos para fluxos com 0,1 L.s ${ }^{-1}(5,28 \pm 0,26 \mathrm{D}, 5,28 \pm 0,36 \mathrm{E}), 0,2 \mathrm{~L} . \mathrm{s}^{-1}$ $(7,75 \pm 0,36 \mathrm{D}, 7,97 \pm 0,21 \mathrm{E})$ e $0,3 \mathrm{~L} \cdot \mathrm{s}^{-1}(10,33 \pm 0,90 \mathrm{D}, 10,76$ $\pm 0,94 \mathrm{E})$, mas foram observadas diferenças significativas para fluxos de $0,5 \mathrm{~L} . \mathrm{s}^{-1}(14,09 \pm 0,49 \mathrm{D}, 14,81 \pm 0,47 \mathrm{E})$ e 0,83 L. ${ }^{-1}(20,04 \pm 0,48 \mathrm{D}, 21,58 \pm 0,04 \mathrm{E})$.

Os fluxos de 0,1 e 0,2 L. s $^{-1}$ resultaram em resistência à passagem em níveis semelhantes para uma mesma sonda de intubação, exceto para cânula de $7 \mathrm{~mm}(p<0,001)$ em que o fluxo de $0,2 \mathrm{~L} . \mathrm{s}^{-1}$ determina maior resistência quando comparado ao fluxo de $0,1 \mathrm{~L} . \mathrm{s}^{-1}$. Na sonda $37 \mathrm{FR}$ com oclusão esquerda ou direita, o fluxo de $0,2 \mathrm{~L} . \mathrm{s}^{-1}$ promove maior resistência quando comparado ao fluxo de $0,1 \mathrm{~L}^{-1} \mathrm{~s}^{-1}(\mathrm{p}<$ $0,001)$. Todas as medidas de resistência para os fluxos acima de $0,2 \mathrm{~L} . \mathrm{S}^{-1}$, para uma mesma cânula, foram significativamente diferentes quando comparados com os fluxos imediatamente inferiores.

\section{DISCUSSÃO}

Nesta investigação, os padrões resistivos da cânulas 37 FR e 7,5 mm mostraram-se bastante próximos. A oclusão de algum ramo da sonda de dupla luz eleva significativamente a resistência, mas de modo indistinto em ambos os ramos para fluxos abaixo de $0,5 \mathrm{~L} . \mathrm{S}^{-1}$.

O uso da sonda tipo $37 \mathrm{FR}$ com os ramos abertos sugere o uso de tempo inspiratório e volume corrente ajustado no ventilador para gerar um fluxo igual ou menor que $0,5 \mathrm{~L} . \mathrm{s}^{-1}$. Tal procedimento faz-se necessário pelo fato de os aparelhos de anestesia habitualmente empregados não disporem de controle para ajuste direto do fluxo. O uso destes valores como referência no ajuste do ventilador pode auxiliar a manter a resistência da cânula próxima aos níveis clinicamente mais utilizados, além de pouco interferir com os fluxos de gases frescos utilizados. Para cálculo do fluxo inspiratório utilizado pode ser observado um exemplo clínico em que um paciente anestesiado recebe um volume corrente de $600 \mathrm{ml}$, com freqüência respiratória de 10 incursões por minuto, relação tempo inspiratório: expiratório de 1:2 e fluxo de gases fres$\cos (\mathrm{FGF})$ de $2 \mathrm{~L} \cdot \mathrm{min}^{-1}\left(0,033 \mathrm{~L} . \mathrm{s}^{-1}\right)$. O volume corrente será acrescido do FGF durante o tempo inspiratório, o que determina nessa situação teórica um volume corrente de $633 \mathrm{mle}$ fluxo de $0,63 \mathrm{~L} . \mathrm{s}^{-1}$. É preciso lembrar que nem todos os aparelhos de anestesia somam o FGF ao volume corrente determinado.

Em levantamento prévio realizado nesta Instituição, a média do volume corrente utilizado situou-se em $600 \mathrm{ml}$, com freqüência respiratória em 10 incursões por minuto e fluxo de gases frescos em $2 \mathrm{~L}$. $\mathrm{min}^{-1}$. Também se observou uma moda para relação de tempo inspiratório e expiratório em 1:2 ${ }^{6}$. Com estes dados, pode-se inferir que o fluxo inspiratório teórico utilizado em aparelhos de anestesia em procedimentos anestésico-cirúrgicos no centro cirúrgico do HCFMUSP situa-se próximo a $38 \mathrm{~L}$. $\mathrm{min}^{-1}$. Para as cânulas testadas, este fluxo inspiratório, se situado próximo ao de $0,5 \mathrm{~L} . \mathrm{s}^{-1}$, gera pa- drões resistivos crescentes quando o diâmetro interno da cânula de intubação é menor.

A oclusão de qualquer ramo pode recomendar redução de fluxos situados acima de $30 \mathrm{~L} \cdot \mathrm{min}^{-1}$. Nessa situação, o padrão resistivo observado $\left(14,09\right.$ ou $\left.14,81 \mathrm{cmH}_{2} \mathrm{O} . \mathrm{L}^{-1} \cdot \mathrm{s}^{-1}\right) \mathrm{si}-$ tuou-se em pelo menos 3 vezes aquele observado para a própria cânula $37 \mathrm{FR}(4,78)$ ou para sonda com diâmetro interno $7,5 \mathrm{~mm}(4,60)$. A despeito de não se observarem diferenças na resistência entre os ramos para fluxos de até 0,3 L. $\mathrm{s}^{-1}$, sua oclusão pode acarretar interferência na resistência total do sistema. Fluxo acima deste considerado pode ocasionar ainda maior interferência.

O filtro impõe resistência quando há associação em série com as sondas de intubação. Essa resistência pode ser limitante para sondas com maior diâmetro ( 8 e $8,5 \mathrm{~mm}$ ) em baixo fluxo $\left(0,1\right.$ e $\left.0,2 \mathrm{~L} . \mathrm{S}^{-1}\right)$, quando a resistência pode ser menor que a observada para o filtro (Tabela I). No entanto, a elevação do fluxo inspiratório não altera o padrão imposto pelos filtros, como observado na figura 1.

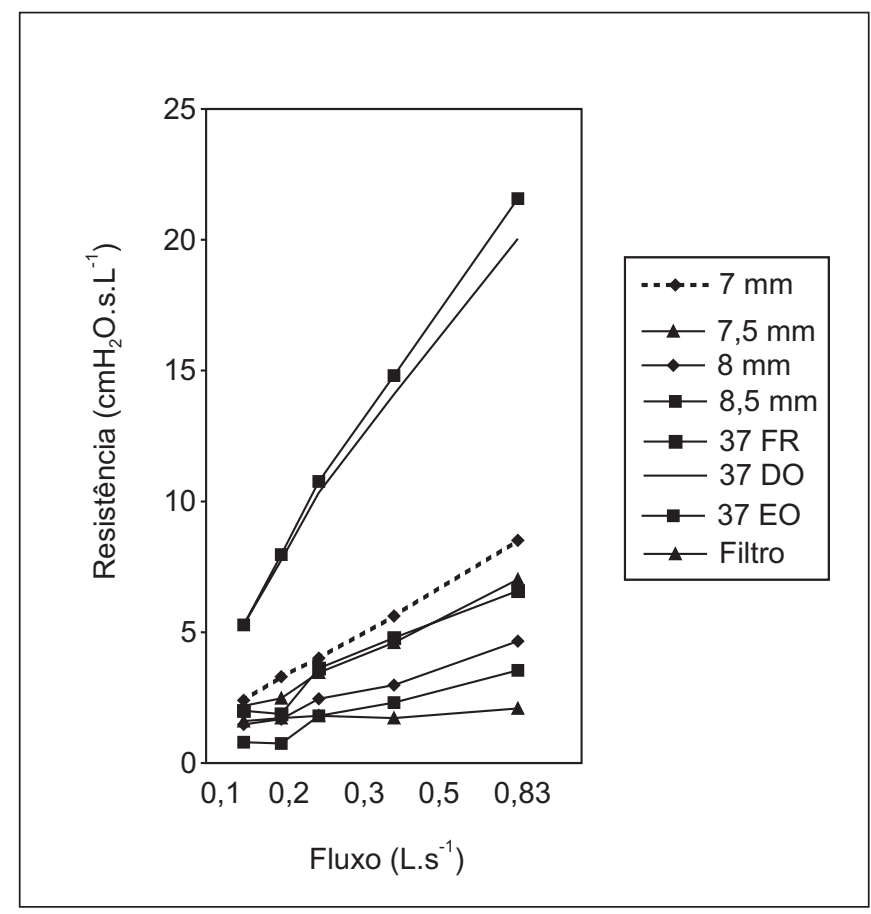

Figura 1 - Resistência Oferecida pelas Cânulas, em cm $\mathrm{C}_{2} \mathrm{O}$ em segundos por litro $\left(\mathrm{cmH}_{2} \mathrm{O} . \mathrm{s} . \mathrm{L}^{-1}\right)$, para os fluxos de $0,1,0,2,0,3$, 0,5 e $0,83 \mathrm{~L} . \mathrm{s}^{-1}$. Maior resistência é vista para sonda de duplo lúmen com ramo ocluído, esquerdo ou direito

Existe a possibilidade das resistências observadas neste experimento mostrarem-se alteradas durante o uso clínico decorrente do acúmulo de umidade. O estreitamento da luz das cânulas pode ser discreto, mas causando eventual interferência nas medidas de resistência. No entanto, neste trabaIho foi utilizado gás fresco, não adicionado de umidade controlada, para determinação da resistência provocada exclusivamente pela sonda.

Nesta investigação não foram medidas as resistências para maiores séries de possíveis fluxos, tampouco foram estuda-

Revista Brasileira de Anestesiologia Vol. 52, N 6, Novembro - Dezembro, 2002 
das as pressões geradas em outras sondas com calibres acima de $8,5 \mathrm{~mm}$ ou abaixo de $7 \mathrm{~mm}$ de diâmetro. Asonda de dupla luz utilizada foi a 37 FR. O motivo para esta limitação decorre do fato destas sondas serem as mais largamente utilizadas em serviços de anestesia. A partir dos dados aferidos, é possível prever que sondas de maior calibre possam oferecer menor resistência. Caso as condições anatômicas ou clínicas permitam, o anestesiologista observará menor resistência ao utilizar sondas de dupla luz com maior diâmetro interno. Por outro lado, sondas menores seguramente impõem maior cuidado no ajuste do fluxo inspiratório para reduzir perda de ventilação pela dissipação da pressão resistiva na cânula.

As resistências observadas neste estudo diferiram do encontrado por Hannallah e col. em investigação utilizando sondas de dupla luz 35, 37, 39 e 41 FR. As resistências encontradas em nosso meio foram maiores para cânulas com diâmetro externo comparável sob fluxos aproximados ${ }^{7}$. No entanto, em nosso estudo não foram observadas diferenças entre os ramos direito e esquerdo da cânula $37 \mathrm{FR}$ para todos os fluxos estudados. Estas diferenças entre as resistências encontradas entre cânulas possivelmente comparáveis podem ser decorrentes de métodos ou sondas utilizadas.

A resistência aumenta para fluxos inspiratórios elevados. $O$ padrão de comparação da sonda de dupla luz com ambos ramos abertos pode ser considerado a resistência oferecida pela cânula com $7,5 \mathrm{~mm}$ de diâmetro interno. O filtro bacteriano acoplado em série com estas sondas tem resistência invariável com fluxo, porém pode aumentar a do sistema de ventilação quando associado a sondas de maior calibre em baixo fluxo. Finalmente, os resultados sugerem a redução de fluxos inspiratórios como parâmetro para minimizar a resistência do sistema de ventilação.

\section{Airflow Resistance of Double-Lumen Tra- cheal Tubes}

Klaus Carvalho Lustosa, M.D., Eric Schalch, M.D., Joaquim Edson Vieira, TSA, M.D., Fábio Ely Martins Benseñor, M.D.

\section{INTRODUCTION}

The progress of current chest surgery is a consequence of the better understanding of open chest patients' physiology. In the early days of the specialty, access to thoracic cavity was the barrier to be overcome, since pneumothorax in a patient under spontaneous ventilation may lead to death ${ }^{1}$. This problem was solved with the introduction of positive pressure mechanical ventilation through single lumen tracheal tubes. Single lung ventilation with double lumen tubes allow for a better access to the thoracic cavity. These tubes help surgical presentation and the surgeon's work, decrease lung trauma and improve the ventilatory assistance of intensive care unit patients. Nevertheless, they also present disadvan- tages, such as tracheal trauma, laryngeal edema, secondary hypoxemia and excessive increase in airflow resistance ${ }^{2,3}$. Robertshaw-type double lumen tubes seem to be less resistant to flow then their counterparts Carlens or White. Moreover, the latter may present different resistances to flow measured in the lateral and lower inlet of their right branch. This disparity may eventually lead to non-homogenous ventilation ${ }^{4}$. In clinical situations, however, it seems to be no flow decrease in currently used double lumen tubes ${ }^{5}$. Bacterial filters are often associated to the use of double lumen tubes, especially with the aim of protecting ventilator branches and monitors against contamination by patients' airways secretion.

This study aimed at comparing the resistive pattern of the 37 FR double lumen tube and conventional tubes used in our country, when submitted to different flows. Resistive properties of the bacterial filter used in tracheal intubation tubes have also been observed.

\section{METHODS}

Tracheal tubes with internal diameters of $7,7.5,8$ and $8.5 \mathrm{~mm}$ and $37 \mathrm{FR}$ double lumen tubes (Mallinckrodt, St. Louis, MO) were tested. Filter was Thermovent BVF 100/584/100 (Portex, UK). Flows were generated and maintained by the anesthesia ventilator Linea (Intermed, São Paulo, Brazil). Resistive pressures were measured by a variable inlet pneumotachograph (Bicore CP100 Respiratory Monitor, Allied Healthcare, USA), connected to the anesthesia machine circle system.

The double lumen tube allows one or both lungs to be ventilated and is used in chest surgeries. The tested tube has the left branch directed to the bronchus and the right branch to the trachea, and is built with the same semi-rigid plastic material used for single lumen tubes.

Flows were previously gaged for a standard reference (Timeter RT200, Allied Healthcare, USA), and remained in $0.1,0.2,0.3,0.5$ and 0.83 liters per second $\left({\mathrm{L} . \mathrm{S}^{-1}}^{-1}\right.$ ) (respectively $6,12,18,30$ and 50 liters per minute). Analog data acquired from the pneumotachograph by a sensor (Var-Flex, Allied Healthcare, USA) were converted to the digital system by an A/V board in a frequency of $200 \mathrm{~Hz}$ and its Aqdados software, and stored in a PC.

Resistances were obtained by dividing measured pressures by flows. Resistances to different flows in similar tubes and to the same flow in different tubes were compared. Data were submitted to analysis of variance (ANOVA) and compared by Tukey's test, considering significant $p<0.05$ between groups, for $\alpha=0.05$ and $\beta=0.80$.

\section{RESULTS}

Resistances were recorded in five random measurements for tubes and flows. All tubes were opened to the environment. 37 FR double lumen tube was tested for both ways, simultaneously, or for each way separately with the lateral 
LUSTOSA, SCHALCH, VIEIRA ET AL

Table I - Resistance to Different Gas Flows through Evaluated Tubes (Mean \pm SD)

\begin{tabular}{lccccc}
\hline \multicolumn{5}{c}{ Flows $\left(\right.$ L.s - $\left.^{-1}\right)$} \\
\hline Tubes & 0.1 & 0.2 & 0.3 & 0.5 & 0.83 \\
$7 \mathrm{~mm}$ & $2.40(0.09)$ & $3.29(0.17)$ & $4.01(0.24)$ & $5.62(0.06)$ & $8.51(0.20)$ \\
$7.5 \mathrm{~mm}$ & $2.18(0.12)$ & $2.47(0.07)$ & $3.45(0.36)$ & $4.60(0.15)$ & $7.02(0.47)$ \\
$8 \mathrm{~mm}$ & $1.47(0.21)$ & $1.68(0.09)$ & $2.46(0.15)$ & $2.98(0.18)$ & $4.66(0.37)$ \\
$8.5 \mathrm{~mm}$ & $0.80(0.17)$ & $0.75(0.08)$ & $1.81(0.17)$ & $2.31(0.39)$ & $3.54(0.23)$ \\
$37 \mathrm{FR}$ & $2.00(0.08)$ & $1.88(0.13)$ & $3.61(0.39)$ & $4.78(0.17)$ & $6.58(0.31)$ \\
$37 \mathrm{DO}$ & $5.28(0.26)$ & $7.75(0.36)$ & $10.33(0.90)$ & $14.09(0.49)$ & $20.04(0.48)$ \\
$37 \mathrm{EO}$ & $5.28(0.36)$ & $7.97(0.21)$ & $10.76(0.94)$ & $14.81(0.47)$ & $21.58(1.04)$ \\
Filter & $1.60(0.06)$ & $1.72(0.11)$ & $1.81(0.09)$ & $1.72(0.05)$ & $2.09(0.09)$ \\
\hline
\end{tabular}

branch occluded. Means and standard deviations of resistances obtained in $\mathrm{cmH}_{2} \mathrm{O} \cdot \mathrm{L}^{-1} \cdot \mathrm{s}^{-1}$ are shown in table I. The 37 FR double lumen tube had resistive patterns similar to the $7.5 \mathrm{~mm}$ tube for all tested flows, however without statistical significance $\left(0.1 \mathrm{~L} . \mathrm{s}^{-1}\right.$ flow $=2.0 \pm 0.08 \mathrm{vs} .2 .18 \pm 0.12 ; 0.2$ L. s $^{-1}$ flow $=1.88 \pm 0.13$ vs. $2.47 \pm 0.07 ; 0.3$ L. $^{-1}$ flow $=3.61 \pm$ 0.39 vs. $3.45 \pm 0.36 ; 0.5{\mathrm{~L} . \mathrm{s}^{-1}}^{-}$flow $=4.78 \pm 0.17$ vs. $4.60 \pm 0.15$; and $0.83{\mathrm{~L} . \mathrm{s}^{-1}}^{-1}$ flow $=6.58 \pm 0.31$ vs. $7.02 \pm 0.47$ ). Resistance is linearly increased with increased flow, but it is decreased in larger internal diameter tubes submitted to the same flow. The occlusion of 37 FR tube branches significantly increases resistance. Filter resistances increased with flow, however without statistical significance. Conversely, the $0.83{\mathrm{~L} . \mathrm{s}^{-1}}^{-1}$ flow imposes significant resistance as compared to those offered by the filter under lower flows $(p<0.001)$.

Branches occlusion has caused a higher resistance increase, but still as a function of flow. There were no significant differences between branches for flows of 0.1 L. ${ }^{-1}(5.28 \pm 0.26 \mathrm{R}, 5.28 \pm 0.36 \mathrm{~L}), 0.2{\mathrm{~L} . \mathrm{s}^{-1}}^{-1}(7.75 \pm 0.36 \mathrm{R}$, $7.97 \pm 0.21 \mathrm{~L})$ and $0.3 \mathrm{~L} . \mathrm{s}^{-1}(10.33 \pm 0.90 \mathrm{R}, 10.76 \pm 0.94 \mathrm{~L})$, but there were significant differences for flows of 0.5 L. S $^{-1}(14.09 \pm 0.49 \mathrm{R}, 14.81 \pm 0.47 \mathrm{~L})$ and $0.83{\mathrm{~L} . \mathrm{S}^{-1}}^{-1}(20.04 \pm$ $0.48 \mathrm{R}, 21.58 \pm 0.04 \mathrm{~L})$.

Flows of 0.1 and 0.2 L.s ${ }^{-1}$ resulted in similar resistances for the same tube, except for the $7 \mathrm{~mm}$ tube $(p<0.001)$, where the $0.2{\mathrm{~L} . \mathrm{S}^{-1}}^{-1}$ flow determined higher resistance as compared to $0.1 \mathrm{~L} . \mathrm{s}^{-1}$ flow. For the $37 \mathrm{RF}$ tube with left or right occlusion, 0.2 L. $\mathrm{s}^{-1}$ flow promoted higher resistance as compared to 0.1 L. $s^{-1}$ flow $(p<0.001)$. All resistance measurements for flows

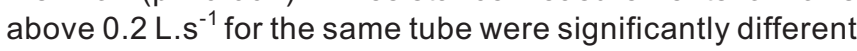
as compared to immediately lower flows.

\section{DISCUSSION}

In our study, resistive patterns of $37 \mathrm{FR}$ and $7.5 \mathrm{~mm}$ tubes were very similar. The occlusion of any double lumen tube branch significantly increases resistance, but in an indistinguishable manner for both branches with flows below 0.5 L. $\mathrm{s}^{-1}$.
The use of 37 FR tube with open branches suggests inspiratory time and tidal volume adjusted to generate a flow equal to or below $0.5 \mathrm{~L} . \mathrm{S}^{-1}$. Such procedure is needed because currently used anesthesia devices do not have controls for direct flow adjustment. The use of these values as a reference to adjust ventilation may help maintain tube resistance closer to more widely used clinical levels, in addition to having a minor influence in fresh gas flows. To calculate the inspiratory flow a clinical example of an anesthetized patient receiving a tidal volume of $600 \mathrm{ml}$, with respiratory rate of 10 incursions per minute, with an inspiratory / expiratory ratio of $1: 2$ and fresh gases flow (FGF) of $2 \mathrm{~L} \cdot \mathrm{min}^{-1}(0.033$ L. $\mathrm{S}^{-1}$ ) may be used. FGF will be added to tidal volume during the inspiratory time determining, in this theoretical situation, a tidal volume of $633 \mathrm{ml}$ and a flow of $0.63 \mathrm{~L} . \mathrm{s}^{-1}$. It must be reminded that not all anesthesia devices add FGF to tidal volume.

In a previous survey performed in our institution, tidal volume mean was $600 \mathrm{ml}$, with respiratory rate of 10 incursions per minute and $2 \mathrm{~L}$. $\mathrm{min}^{-1}$ fresh gas flow. An inspiratory/expiratory ratio mode of $1: 2$ was also observed ${ }^{6}$. With such data, it is possible to assume that the theoretical inspiratory flow used in anesthesia machines for surgical-anesthetic procedures in the operating center, HCFMUSP, is approximately to 38 L. $\mathrm{min}^{-1}$. For the tested tubes, this inspiratory flow, if close to $0.5 \mathrm{~L} . \mathrm{s}^{-1}$, generates increasing resistive patterns when the internal diameter of the tube is smaller.

The occlusion of any branch may recommend the decrease of flows above $30 \mathrm{~L} \cdot \mathrm{min}^{-1}$. In these situations, the observed resistive pattern (14.09 or $14.81 \mathrm{cmH}_{2} \mathrm{O} \cdot \mathrm{L}^{-1} \cdot \mathrm{s}^{-1}$ ) was at least three times higher than that observed for the same $37 \mathrm{FR}$ tube (4.78) or for the $7.5 \mathrm{~mm}$ tube (4.60). Although no differences in resistance observed between branches for flows up to 0.3 L.S ${ }^{-1}$, their occlusion may lead to interferences in the system's total resistance. Flows higher than this would interfere even more.

The filter imposes resistance when there is a serial association with tracheal tubes. This resistance may be limiting for larger tubes ( 8 and $8.5 \mathrm{~mm}$ ) and low flows $\left(0.1\right.$ and $0.2{\mathrm{~L} . \mathrm{s}^{-1}}^{-1}$ ), situation in which the resistance may be lower than for the filter (Table I). The increase in inspiratory flow, however, does

Revista Brasileira de Anestesiologia Vol. 52, № 6, Novembro - Dezembro, 2002 
not change the patterns imposed by the filters, as shown in figure 1.

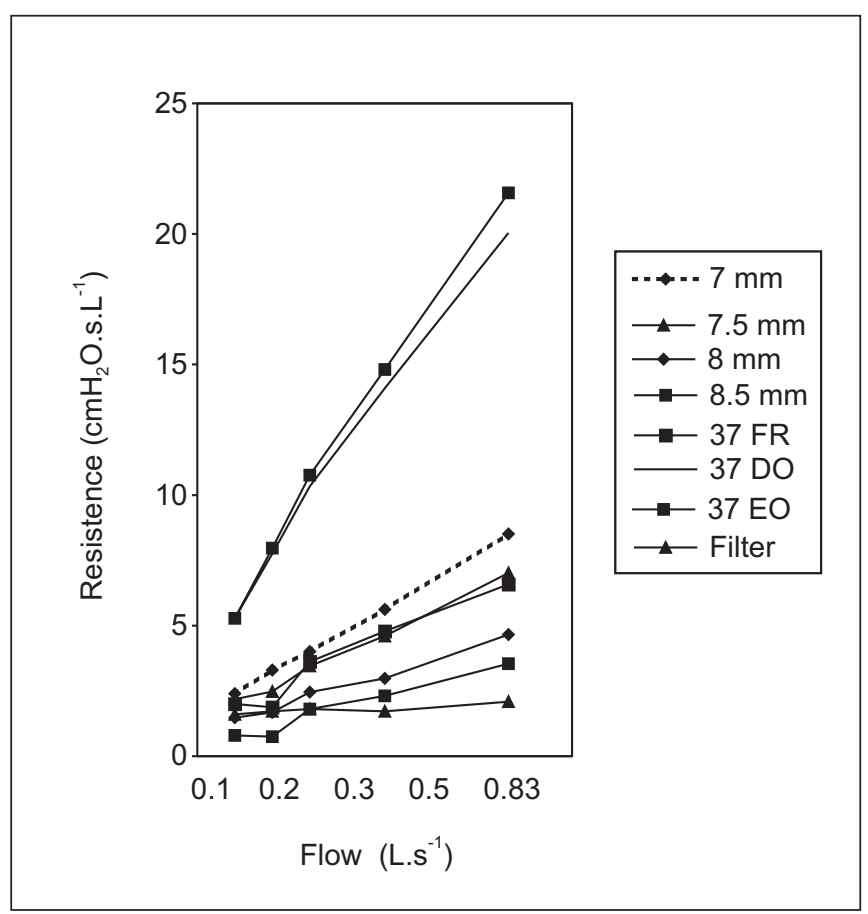

Figure 1 - Tubes Resistance in $\mathrm{cmH}_{2} \mathrm{O}$ in seconds per liter $\left(\mathrm{cmH}_{2} \mathrm{O} . \mathrm{s.L}^{-1}\right)$, for $0.1,0.2,0.3,0.5$ and $0.83{\mathrm{~L} . \mathrm{s}^{-1}}^{-1}$ Flows. Higher resistance is seen for the double lumen tube with right or left occluded branch

It is possible that the resistances observed in our study may be changed by excess moisture, during clinical use. The narrowing of tube lumen may be minor, but nevertheless interferes with resistance measurements. In our study, fresh gas without controlled moisture was used to determine the resistance caused exclusively by the tube.

Our study has not measured resistances for the highest possible series of flows or pressures generated in other tubes larger than $8.5 \mathrm{~mm}$ or smaller than $7 \mathrm{~mm}$. Double lumen tube was the $37 \mathrm{FR}$. This limitation was a consequence of these being the most widely used tubes in anesthesiology departments. As from our data, it is possible to assume that larger tubes may offer less resistance. If allowed by anatomical or clinical conditions, the anesthesiologist should observe less resistance with larger double lumen tubes. On the other hand, smaller tubes clearly ask for more attention in adjusting inspiratory flow to decrease ventilation loss by resistive pressure dissipation in the tube.

Resistances observed in this study were different from was has been found by Hannallah et al. in a study using 35, 37, 39 and $41 \mathrm{FR}$ double lumen tubes. Our resistances were higher for tubes with comparable diameters under approximate flows ${ }^{7}$. However, we have not observed differences between 37 FR tube right and left branches for all studied flows. Resistance differences in comparable tubes may be a function of methods or tubes used.

Revista Brasileira de Anestesiologia

Vol. 52, N 6 , Novembro - Dezembro, 2002
Resistance increases for high inspiratory flows. The comparison pattern of the double lumen tube with both branches open may be considered the resistance offered by the 7.5 $\mathrm{mm}$ tube. The bacterial filter coupled in series with these tubes has a resistance which does not change with flow, but may increase ventilation system resistance if associated to larger tubes and low flows. Last, but not least, our results suggest the decrease in inspiratory flow as the parameter to minimize ventilation system resistance.

\section{REFERÊNCIAS - REFERENCES}

01. Ferez D - Anestesia para Cirurgia Torácica, em: Ortenzi VO, Tardelli MA - Anestesiologia SAESP, São Paulo, Atheneu, 1996;647-671.

02. Heck JR - Anestesia em Cirurgia Torácica, em: Manica JD Anestesiologia. Princípios e Técnicas, Porto Alegre, Artes Médicas, 1997;452-467.

03. Tobin MJ - Monitoring of Lung Mechanics and Work of Breathing, em: Tobin MJ. - Principles and Practice of Mechanical Ventilation, New York, McGraw-Hill, 1994;969-1056.

04. Hammond JE, Wright DJ - Comparison of the resistances of double-lumen endobronchial tubes. Br J Anaesth, 1984;56: 299-302.

05. Slinger PD, Lesiuk L - Flow resistances of disposable double-lumen, single-lumen, and univent tubes. J Cardiothorac Vasc Anesth, 1998;12:142-144.

06. Silva BAR, Garcia Jr D, Vieira JE - Ventilação mecânica em procedimentos anestésico-cirúrgicos. Rev Bras Anestesiol, 2001;51:(Supl27):CBA135A.

07. Hannallah MS, Miller SC, Kurzer SI et al - The effective diameter and airflow resistance of the individual lumens of left polyvinyl chloride double-lumen endobronchial tubes. Anesth Analg, 1996;82:867-869.

\section{RESUMEN}

Lustosa KC, Schalch E, Vieira JE, Benseñor FEM - Resistencia al Flujo de Gases a través de las Cánulas de Intubación de Dupla Luz

Justificativa y Objetivos - Las cánulas de dupla luz pueden imponer distintas resistencias al flujo. Esta disparidad puede ocasionar ventilación pulmonar no homogénea. El objetivo de este trabajo es comparar el patrón resistivo de la cánula de intubación de dupla luz 37 FR con las cánulas convencionales, utilizadas en nuestro medio, cuando sometidas a flujos diferentes.

Método - Fueron utilizadas cánulas de intubación orotraqueal con diámetros internos de 7, 7,5, 8 y 8,5 mm y de dupla luz 37 $F R$. Los flujos fueron generados y mantenidos por aparato de anestesia. La presión resistiva generada en las cánulas fue aferida por un pneumotacógrafo de orificio variable y convertida para sistema digital. Las resistencias fueron obtenidas dividiéndose las presiones medidas por los flujos empleados. Los datos fueron sometidos a un análisis de variancia (ANOVA) y comparados por el test de Tukey.

Resultados - Fueron registradas cinco medidas obtenidas aleatoriamente. Todas las cánulas estaban abiertas para el medio-ambiente. Resistencias están en $\mathrm{cmH}_{2} \mathrm{O} \cdot \mathrm{L}^{-1} . \mathrm{s}^{-1}$. La resistencia aumenta de forma linear con elevación del flujo. La cánula 37 FR dupla luz mostró patrón resistivo semejante al de la cánula con diámetro interno de $7,5 \mathrm{~mm}$. La oclusión de los 
ramos de la sonda 37 FR eleva significativamente la resistencia al flujo. Los flujos de 0,1 a $0,2 \mathrm{~L}_{\text {. }} \mathrm{s}^{-1}$ resultaron en presiones resistivas semejantes para una misma sonda de intubación, excepto para cánula $7 \mathrm{~mm}$ o para la $37 \mathrm{FR}$ con oclusión $(p<0,001)$.

Conclusiones - Los patrones resistivos de las cánulas 37 FR y $7,5 \mathrm{~mm}$ se mostraron bastante próximos. La oclusión de algún ramo de la sonda de dupla luz eleva significativamente la resistencia, más de modo indistinto entre ambos ramos para

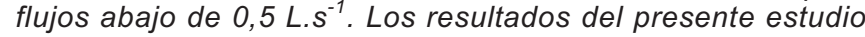
sugieren la reducción de flujos inspiratorios como parámetro para disminuir la resistencia del sistema de ventilación, cuando uno de los lúmenes de un tubo de dupla luz es obstruido. 